\title{
Nanostructured exchange coupled hard / soft composites: from the local magnetization profile to an extended 3D simple model
}

\author{
V. Russier, K. Younsi and L. Bessais \\ ICMPE, UMR 7182 CNRS and University UPEC, \\ 2 rue Henri Dunant, 94320 Thiais, France.
}

\begin{abstract}
In nanocomposite magnetic materials the exchange coupling between phases plays a central role in the determination of the extrinsic magnetic properties of the material: coercive field, remanence magnetization. Exchange coupling is therefore of crucial importance in composite systems made of magnetically hard and soft grains or in partially crystallized media including nanosized crystallites in a soft matrix. It has been shown also to be a key point in the control of stratified hard / soft media coercive field in the research for optimized recording media. A signature of the exchange coupling due to the nanostructure is generally obtained on the magnetization curve $M(H)$ with a plateau characteristic of the domain wall compression at the hard/soft interface ending at the depinning of the wall inside the hard phase. This compression / depinning behavior is clearly evidenced through one dimensional description of the interface, which is rigorously possible only in stratified media. Starting from a local description of the hard/soft interface in a model for nanocomposite system we show that one can extend this kind of behavior for system of hard crystallites embedded in a soft matrix.
\end{abstract}

\section{INTRODUCTION}

A simple model in order to understand the relation between the nanoscale structure and the extrinsic magnetization curves in partly crystallized rare earth transition metal alloys (Re-M), can be built as an assembly of nanosized crystallites embedded in an amorphous matrix. Such a model is based upon experimental caracterization of the samples obtained from an amorphous precursior, obtained either by high energy milling or a melt spun technique for instance, followed by an appropriate annealing which induces the re-crystallization [1 4$]$. The crystallites and the matrix must be characterized by hard and soft magnetocrystalline anisotropy respectively. One key point concerning the extrinsic magnetic properties of the system is then the exchange coupling between the crystallites and the matrix or between the crystallites via the matrix. In other words, since one deals with nanocomposite including magnetic hard and soft phases, such models can enter in the so-called exchange spring magnet (ES) systems [5-11]. Exchange coupling between different phases of nanostructred composite materials have been widely studied after the pioneering work of Aharoni [12] mainly through the behavior of the average magnetization curve in terms on the applied field, $M(H)$ [4, 10, 13 17]. On the other hand the nucleation field corresponding to the very beginning of the magnetization reversal in the soft phase has been determined semi-analytically in different situations from the linearization of the micromagnetic energy with respect to the deviation of the local magnetization from the direction imposed by the hard phase easy axis [14, 16, 18, 19]. Conversely, the local magnetization $\mathbf{m}(\mathbf{r})$ in the vicinity of the interface between phases in nanocomposites has not been investigated in details but in continuous film geometry (layered systems) 20 22], where furthermore the effect of the hard and soft layers thickness has been investigated through the magnetic phase diagram representing the reversal mode of the demagnetization, namely rigid mode 
versus ES-coupled mode, [13 16] via the analytical determination of the magnetic susceptibility. In the works devoted to the magnetization reversal in ES layered systems through the behavior of the local magnetization in terms of the distance, $z$, normal to the interface the solvation of the Euler equations relative to the micromagnetic energy minimization is made tractable because of the symmetry parallel to the surface according to which the local magnetization depend only on $z$ 20 22]. This makes the nucleation, domain wall (DW) compression and depinning process at the hard / soft interface a well established theoretical result [6, 9, 17, 21] which moreover has been experimentally observed [6, 23, 24]. In the case of nanocomposites made of true 3D grains either simply juxtaposed or separated by grain boundaries, the situation is not so clear and as a general rule one refers to the similarity in the $M(H)$ curve to map the magnetization reversal process to the one indeed obtained for the ES layered system.

In the present work, we deal with a model made of hard inclusions embedded in a soft matrix with the purpose to exploit the above mentioned theoretical results on the magnetization reversal in magnetically hard/soft composite systems. More precisely our aim is to bridge the gap between the stratified media, for which the reversal process is well understood, and our system of magnetically hard material inclusions embedded in the soft matrix. We investigate the way according to which one can map the well-known nucleation, DW compression and depinning process of the magnetization reversal characteristic of the exchange coupled stratified media to the exchange coupled inclusion / matrix system. We will use both analytical results for the angle profile at the planar interface in a one dimensional approximation and numerical results for true 3-D systems obtained from a micromagnetism finite element based code (MAGPAR) [25].

In a first step, we compare the 1-D profile to the simulated result for one cubic inclusion embedded in a prismatic matrix. The easy axis of the inclusion is parallel to the interface (parallel recording media type of geometry). The variation of $M(H)$ in the plateau region characteristic of layered ES media is very well reproduced, and the accuracy obtained for the magnetization profile, shows that the nucleation, domain wall compression and depinning process is valid for isolated inclusions embedded in a very soft matrix.

In order to investigate the effect of the finite value taken by the edge to edge separation $\Delta$ between the crystallites in the actual system, we consider a model made of two cubic inclusions in the soft matrix. The magnetization profile behavior in terms of the applied field shows that the magnetization reversal follows the nucleation, domain wall compression and depinning process when $\Delta$ is larger than the exchange length $l_{e x}$ of the soft material whereas when $\Delta$ gets smaller than $l_{e x}$ the magnetization reverses in a coherent way in the whole system which corresponds to the strong coupling regime, or the rigid composite magnet of Refs. [13, 14], where the system behaves as a single phase.

Then, we consider the effect of the misallignment between the easy axes of the neighboring inclusions. In this case, the domain wall which was of Bloch type for the parallel oriented interface, takes clearly a Néel character, with nevertheless at the qualitative level, the same type of magnetization reversal processes. 


\section{ONE DIMENSIONAL MODEL}

In this section, we first recall the basic equations concerning the one dimensional description for the magnetization profile accross the hard soft interface. We consider the planar sharp interface between a uniaxial hard material and an ultra soft material. The interface, normal to the $\hat{z}$ axis, is defined by $z=z_{0}$ and the easy axis of the hard material, this latter being located at $z>z_{0}$, coincides with the $\hat{x}$ axis. The micromagnetic characteristics of the media will be denoted $K_{s, h}, A_{s, h}, J_{s, h}$ where the subscript $s(h)$ refers to the soft (hard) phase and $K, A, J$ denote the magnetocrystalline anisotropy constant, the exchange stiffness and $\mu_{0}$ times the saturation magnetization respectively. Here we shall consider the limiting case of an ultra soft phase, with $K_{s}=0$. Then the properties of the one dimensional model are totally determined by the reduced parameters $\epsilon_{A}=A_{s} / A_{h}$, $\epsilon_{J}=J_{s} / J_{h}$, the reduced distance $z *=z / \delta_{B}, \delta_{B}$ being the Bloch DW thickness in the hard phase, $\delta_{B}=\pi \sqrt{A_{h} / K_{h}}$ and the reduced field $h=-H_{a} / H_{K}$ where $H_{a}$ and $H_{K}=2 K_{h} / J_{h}$ are the applied and the hard-phase anisotropy field respectively. The minus sign in the definition of $h$ is only for convenience in order to deal with $h>0$ in the second quadrant $H_{a}<0$. We consider the case of an external field $\mathbf{H}_{a}$ in the $\hat{x}$ direction. Starting from the energy functional where we explicitly assume the magnetization profile to lie in the plane $(\hat{x}, \hat{y})$ and to depend only on $z$

$$
\begin{aligned}
E[\hat{m}(z)] & =S \int\left[A(z)\left(\left(\frac{\partial m_{x}(z)}{\partial z}\right)^{2}+\left(\frac{\partial m_{y}(z)}{\partial z}\right)^{2}\right)+K(z)\left(1-m_{x}(z)^{2}\right)-J(z) m_{x}(z) H_{a}\right] d z \\
X(z) & =X_{s} \Theta\left(z_{0}-z\right)+X_{h} \Theta\left(z-z_{0}\right) ; \quad X=K, A \text { or } J .
\end{aligned}
$$

where $\Theta(z)$ is the Heavyside step function. The minimization of the functional $E[\hat{m}(z)]$ with respect to $\hat{m}(z)$ leads to the well known Euler equations [13, 14, 20 22] which, written in terms of the angular profile $\varphi(z)=(\hat{m}(z), \hat{x})$ reads

$$
\begin{aligned}
\frac{\partial \varphi(z)}{\partial z} & = \pm \frac{1}{\sqrt{A_{h, s}}}\left[\Delta U_{h, s}(\varphi(z))\right]^{1 / 2} \\
\text { with } \Delta U_{h, s}(\varphi(z)) & =K(z)\left(\sin ^{2}(\varphi(z))-\sin ^{2}\left(\varphi\left(z_{b}^{(h, s)}\right)\right)\right)-J(z) H_{a}\left(\cos (\varphi(z))-\cos \left(\varphi\left(z_{b}^{(h, s)}\right)\right)\right)
\end{aligned}
$$

The singularity of the interface leads to the boundary condition at $z=z_{0}$

$$
\left.\left.\epsilon_{A} \frac{\partial \varphi}{\partial z}\right]_{z_{0}^{-}}=\frac{\partial \varphi}{\partial z}\right]_{z_{0}^{+}}
$$

In equation (2) the choice for the \pm sign depends on the boundary conditions far from the interface, represented by the values taken by $\varphi_{b}^{(s, h)}=\varphi\left(z_{b}^{s, h}\right)$ where $z_{b}^{(s, h)}$ denote the location of either the soft or the hard layer center. In the case of the hard / soft interface with $z_{b}^{(s, h)} \rightarrow \pm \infty$ we shall asume in equation (2) that far from the interface, $z \sim z_{b}$, the magnetization $\hat{m}$ is alligned to the easy axis and that either $K_{h, s}>0$ or $K_{h}>0$ and $K_{s}=0\left(\sin \varphi_{b}=0\right)$. Starting from an external field applied in the $x>0$ direction, where $\hat{m}(z)=\hat{x}$ in the whole system, the field is decreased and then the first step in the magnetization reversal is the so-called nucleation field $h=h_{\text {nucl }}$ corresponding to the reversal in the soft phase. $h_{\text {nucl }}$ can be obtained from the expansion of $E[\hat{m}(z)]$ in terms of the small deviations $\delta \vec{m}=(\hat{m}(z)-\hat{x})$ at second order in $|\delta \vec{m}|[18]$. In the 
following we limit ourselves to a hard layer thick enough for $z_{b}^{h}$ to be considered as infinite. Let us first consider the case $z_{b}^{(s)} \rightarrow \infty$. As usual, we consider the situation where the magnetization in the soft phase, far from the interface is along the field, namely $\hat{m}\left(z \sim z_{b}^{(s)}\right)=-\hat{x}$. The profile $\varphi(z)$ can be easyly obtained from the numerical inversion of

$$
z-z_{0}=\int_{\varphi_{0}}^{\varphi(z)} \frac{\partial z}{\partial \varphi} d \varphi=-\int_{\varphi_{0}}^{\varphi(z)} \sqrt{A(z)}\left[\Delta U_{h, s}(\varphi)\right]^{-1 / 2} d \varphi
$$

which can be integrated, with the result

$$
\begin{aligned}
& \left.z^{*}-z_{0}^{*}=\frac{\sqrt{\epsilon_{A} / \epsilon_{J}}}{\pi} \ln \left(\frac{1-\tan (\varphi / 4)}{1+\tan (\varphi / 4)}\right)\right]_{\varphi_{0}}^{\varphi(z)} ; \text { for } \varphi>\varphi_{0} \\
& \left.z^{*}-z_{0}^{*}=-\frac{1}{2 \pi \sqrt{1-h}} \ln \left(\frac{\Delta-\cos (\varphi / 2)}{\Delta+\cos (\varphi / 2)}\right)\right]_{\varphi_{0}}^{\varphi(z)} \text { with } \Delta=\sqrt{1-\frac{\sin ^{2}(\varphi / 2)}{1-h}} ; \text { for } \varphi<\varphi_{0}
\end{aligned}
$$

Here we implicitly asumed that the profile $\varphi(z)$ is a monotonous function of $z$ and accordingly with $\varphi_{b}^{(s, h)}=\pi, 0$ the soft $\left(z<z_{0}\right)$ and the hard $\left(z>z_{0}\right)$ phases correspond to $\pi>\varphi(z)>\varphi_{0}$ and $\varphi_{0}>\varphi(z)>0$ respectively. The value $\varphi_{0}$ of $\varphi$ at $z=z_{0}$ is obtained from the boundary condition (3) [21]

$$
\begin{aligned}
\cos \left(\varphi_{0}\right) & =\frac{1}{\left(1-\epsilon_{A} \epsilon_{K}\right)}\left[h\left(1-\epsilon_{A} \epsilon_{J}\right)\right. \\
& \left.+\left[h^{2}\left(1-\epsilon_{A} \epsilon_{J}\right)^{2}+\left(1-\epsilon_{A} \epsilon_{K}\right)\left[\left(1-\epsilon_{A} \epsilon_{K}\right)-2 h\left(1+\epsilon_{A} \epsilon_{J}\right)\right]\right]^{1 / 2}\right]
\end{aligned}
$$

which is defined up to a critical value which defines the depinning field, i.e. the largest value of $\varphi_{0}$ which can be accomodated by the hard phase, and coincides with the coercive field $h_{c}$ since we expect the nucleation field of the soft phase to be smaller. $h_{c}$ is given by [21]

$$
h_{c}=\frac{1-\epsilon_{A} \epsilon_{K}}{\left(1+\sqrt{\epsilon_{A} \epsilon_{J}}\right)^{2}}
$$

When $z_{b}^{(s)}$ takes a finite value and then coincides with the half thickness of the soft layer, $\varphi_{b}=$ $\varphi_{b}\left(z_{b}^{(s)}\right)$ in equation (2) is considered as a parameter. The profile in equation (4) is numerically integrated and $\varphi_{b}$ is determined from the fulfillment of

$$
\begin{aligned}
z_{b}^{(s)}-z_{0} & =-\int_{\varphi_{0}}^{\varphi_{b}} \sqrt{A_{s}}\left[\Delta U_{s}\left(\varphi, \varphi_{b}\right)\right]^{-1 / 2} d \varphi \equiv I\left(\varphi_{b}\right) \\
\text { with } \cos \left(\varphi_{0}\right) & =h\left(1-\epsilon_{A} \epsilon_{J}\right)+\left[h^{2}\left(\epsilon_{A} \epsilon_{J}-1\right)^{2}+1-2 h\left(1-\epsilon_{A} \epsilon_{J} \cos \varphi_{b}\right)\right]^{1 / 2}
\end{aligned}
$$

from a Newton-Raphson procedure by solving: $I\left(\varphi_{b}\right)-\left(z_{b}^{(s)}-z_{0}\right)=0$.

Let us now consider the case where the easy axis of the hard inclusion is not oriented parallel to the interface, $(\hat{n}, \hat{z})=\theta_{h} \neq \pi / 2$, still with $(\hat{n}, \hat{y})=\pi / 2$. As a general rule, for $\left(\theta_{h}-\pi / 2\right)$ not too small, the numerical simulations lead to a Néel domain wall at the interface, and accordingly we consider only this situation in the one dimensional model. The magnetization is therefore in the $(\hat{x}, \hat{z})$ plane and is totally determined by the angle $\varphi(z)=(\hat{m}, \hat{z})$. As a consequence of the 
longitudinal nature of the domain wall at the soft / hard interface the demagnetizing field in the soft phase must be introduced. We still do not introduce the demagnetizing field in the hard phase, since we aim in fine to model a system where this latter concerns a cubic inclusion characterized by $N_{x}=N_{y}=N_{z}$. The soft phase is enclosed in an ellongated shaped prism, whose long axis, $\hat{z}$, is normal to the interface. In the following we shall consider the 1-D model in the infinitelly long prism geometry with demagnetizing coefficients $N_{x}=N_{y}=1 / 2 ; N_{z}=0$. In the bulk soft phase and if $z_{b}^{(s)} \rightarrow \infty$, the equilibrium value $\varphi\left(z_{b}^{(s)}\right)$ is determined from the minimum of the energy density

$$
E_{b}=-J_{s} H_{a} \sin \left(\varphi_{b}^{(s)}\right)+\frac{1}{2} \Delta N \frac{J_{s}^{2}}{\mu_{0}} \sin ^{2}\left(\varphi_{b}^{(s)}\right) ; \quad \text { with } \Delta N=\left(N_{x}-N_{z}\right)=\frac{1}{2}
$$

with the result

$$
\sin \left(\varphi_{b}^{(s)}\right)=-\inf \left(1, \frac{k h}{\epsilon_{J}}\right) ; \quad \text { with }: H_{a}=-H_{K} h
$$

In the bulk hard phase, the equilibrium value, $\varphi_{b}^{(h)}$ of $\varphi(z)$ is determined in a similar way and can expanded in the vicinity of $\theta_{h}$ because of the high value of $H_{K}$ and we get

$$
\varphi_{b}^{(h)}=\theta_{h}-\frac{h \cos \theta_{h}}{1+h \sin \theta_{h}}
$$

In the present geometry, $\Delta U_{h, s}(\varphi(z))$ are now given by

$$
\begin{aligned}
& \Delta U_{h}(\varphi(z))=A_{h}\left(\frac{\pi}{\delta_{B}}\right)^{2}\left(\sin ^{2}\left(\theta_{h}-\varphi(z)\right)+2 h\left(\sin (\varphi(z))-\sin \left(\varphi_{b}^{(h)}\right)\right)\right) \\
& \Delta U_{s}(\varphi(z))=A_{s}\left(\frac{\pi}{\delta_{B}}\right)^{2} \frac{\epsilon_{J}^{2}}{k \epsilon_{A}}\left(\sin ^{2}(\varphi(z))+\frac{2 k h}{\epsilon_{J}} \sin (\varphi(z))-\sin ^{2}\left(\varphi_{b}^{(s)}\right)-\frac{2 k h}{\epsilon_{J}} \sin \left(\varphi_{b}^{(s)}\right)\right)
\end{aligned}
$$

where we have introduced the hardness factor $k=2 \mu_{0} H_{K} / J_{h}$. In a similar way to what have been done for the parallel oriented interface we get the profile $\varphi(z)$ from the solvation of

$$
z^{*}-z_{0}^{*}=\frac{1}{\pi} \frac{\left(\epsilon_{A} k\right)^{1 / 2}}{\epsilon_{J}} \int_{\varphi(z)}^{\varphi_{0}} \frac{d \varphi}{\left(\sin ^{2}(\varphi)+\left(2 k h / \epsilon_{J}\right) \sin (\varphi)-\sin ^{2}\left(\varphi_{b}^{(s)}\right)-\left(2 k h / \epsilon_{J}\right) \sin \left(\varphi_{b}^{(s)}\right)\right)^{1 / 2}}
$$

in the soft phase and

$$
z^{*}-z_{0}^{*}=\frac{1}{\pi} \int_{\varphi_{0}}^{\varphi(z)} \frac{d \varphi}{\left(\sin ^{2}(\varphi-\theta)+2 h(\sin (\varphi)-\sin (\theta))\right)^{1 / 2}}
$$

in the hard phase. The value $\varphi_{0}$ of $\varphi\left(z=z_{0}\right)$ is determined from the boundary condition and 
results from the numerical solvation of

$$
\begin{array}{r}
\frac{\epsilon_{A} \epsilon_{J}^{2}}{k}\left[\sin \left(\varphi_{0}\right)^{2}+2 h\left(k / \epsilon_{J}\right) \sin \left(\varphi_{0}\right)-\sin \left(\varphi_{b}^{(s)}\right)^{2}-2 h\left(k / \epsilon_{J}\right) \sin \left(\varphi_{b}^{(s)}\right)\right] \\
-\left[\sin \left(\varphi_{0}-\theta_{h}\right)^{2}-\sin \left(\varphi_{b}^{(h)}-\theta_{h}\right)^{2}+2 h\left(\sin \varphi_{0}-\sin \varphi_{b}^{(h)}\right)\right]
\end{array}
$$

As in the preceding case, when $z_{b}^{(s)}$ takes a constant value, the value of $\varphi_{b}^{(s)}$ is no more taken from the equilibrium condition (10) but is determined from the fulfillment of $z\left(\varphi_{b}^{(s)}\right)=z_{b}^{(s)}$ with $z\left(\varphi_{b}^{(s)}\right)$ numerically calculated from equation (13).

Furthermore, we also introduce a way to fit the value of the field, say $h^{(f i t)}$, at which the 1-D profile is calculated in order to enhance the accuracy with the 3-D simulated profile: instead of using the actual value of $h$, we fix the value of $\varphi_{b}^{(s)}$ to that obtained in the simulation. Hence, $h^{(f i t)}$ satisfies

$$
\varphi_{b}^{(s)}\left(h^{(f i t)}\right)=\tilde{\varphi}_{b}^{(s)}(h)
$$

$\tilde{\varphi}_{b}$ being the 3-D micromagnetic simulation result for $\varphi_{b}$.

The angular profile across the interface can be used to understand the behavior of the demagnetization process in exchange coupled media. This has been done in different situations in the literature $66,16,17,26]$, especially in the framework of recording media optimisation although the magnetization profile accross the interface is generally not explicited. Here, our purpose is to make the link with the situation of a lattice of magnetically hard inclusions in a magnetically soft matrix. More precisely, we now compare the $\varphi(z)$ profile calculated from equations (5), (13) 14) with the one extracted from the 3-D micromagnetic simulation in two simple situations.

\section{FEM SIMULATION OF THE GRAIN / MATRIX INTERFACE}

The numerical simulation of the magnetization in terms of the applied field $H_{a}$ is performed by the micromagnetic code MAGPAR [25], based upon a finite elements numerical scheme. The embedded hard grain is a cube of edge length $a=2 R$, with $R=17 \mathrm{~nm}$ being the length scale fixed at a convenient value for nanostructured rare earth - transition metal intermetallics [1 4 ]. We have chosen to keep fixed the magnetic parameters of the hard phase and consequently the Bloch domain wall thickness, $\delta_{B}$ takes a constant value, $\delta_{B}=5 \mathrm{~nm}$ (see section IV below). In the model including only one such embedded grain in order to represent the case of isolated crystallites in the soft matrix this latter is a parallepipedic prism of total length $L_{z}=6 R$, and lateral width $L_{x}=L_{y}=(2 R+\delta)$, with $\delta=0.40 \delta_{B}$ and the embedded grain is located at its center. In the model with two crystallites introduced in order to study the influence of $\Delta / l_{e x}$ on the magnetization profile, we use a fixed edge to edge distance $\Delta=2 \delta_{B}$ and the ratio $\Delta / l_{e x}$ is varied through the value of $l_{e x}=\sqrt{2 \mu_{0} A_{s} / J_{s}^{2}} \equiv \delta_{B} / \pi \sqrt{\mu_{0}\left(H_{K} / J_{h}\right)\left(\epsilon_{A} / \epsilon_{J}^{2}\right)}$ as a function of $\epsilon_{A}$ and $\epsilon_{J}$. The local magnetization profile is extracted along a line parallel to the $\hat{z}$ direction, cutting the grain / matrix interface at its center. A particular attention is paid to the quality of the mesh, which must be superior to what is necessary for the average magnetization curve, $M\left(H_{a}\right)$ over the whole system. Here the quality of the mesh has been controlled through the fulfillment of the unitary condition 
of $\mathbf{m}(\mathbf{r})$ which is exactly satisfied only on the nodes. The typical size of the mesh tetraedra is sharply peaked at $0.10 \delta_{B}$, the maximum edge length for more than half of the tetraedra is less than $0.20 \delta_{B}$ leading to a mesh for our $(2 R+\delta) \times(2 R+\delta) \times 6 R$ model including about $8.510^{5}$ finite elements.

Before going further we have to note that because in our system the soft phase surrounds the whole hard grain, we have to take into account not only the interface we are interested in, normal to $\hat{z}$, but also the other sides of the embedded cubic grain. By symmetry, only the top and bottom ones are to be considered. This is important especially for the nucleation field determination. To estimate the effect of the additional interface, we divide the soft phase domain into the prism based on the side normal to $\hat{z}$ located at $z=z_{0}$ namely bounded by $(x, y)= \pm R$, and the top and bottom layers $(|x|>R)$ for which the hard/soft interface is perpendicularly oriented. For these additional layers, the demagetizing factors are $N_{y}=N_{z} \simeq 0$ and $N_{x} \simeq 1$ and we must add to the energy density a term due to the corresponding demagnetizing field and proportional to the volumic fraction say $\alpha$ occupied by these layers in the total soft phase domain. Then, in the case of the Bloch wall parallel to the interface, the implicit equation from which $h_{n u c l}$ is obtained [14, 15, 18],

$$
\lambda_{h} \tanh \left(\lambda_{h} L_{h} / 2\right)=\epsilon_{A} \lambda_{s} \tan \left(\lambda_{s} L_{s} / 2\right)
$$

still holds but with modified definitions of the parameters $\lambda_{h}$ and $\lambda_{s}$

$$
\begin{aligned}
\lambda_{h}^{2} & =\left(\frac{\pi}{\delta_{B}}\right)^{2}[1-h] \\
\lambda_{s}^{2} & =\left(\frac{\pi}{\delta_{B}}\right)^{2}\left(\frac{\epsilon_{J}}{\epsilon_{A}}\right)\left[h+\alpha \frac{N_{x} J_{s}}{\mu_{0} H_{K}}-\epsilon_{K} / \epsilon_{J}\right]
\end{aligned}
$$

Since $\lambda_{h} L_{h}>1$ for $L_{h}>\delta_{B}$ the value of $h_{n u c l}$ is nearly independent of $L_{h}$, and therefore, we can estimate the effect of the surrounding layers by solving equation (17) for the field $h^{\prime} \simeq$ $h+\alpha N_{z} J_{s} / \mu_{0} H_{K}$. The nucleation field is thus approximately given by $h_{n u c l}=h_{0}-\alpha N_{z} J_{s} / \mu_{0} H_{K}$ where $h_{0}$ is the solution of equ. (17) with $\alpha=0$, and may be negative corresponding to a nucleation of the soft phase in the first quadrant. For the same geometrical reason, we do not expect the coercive field to be given by the analytical result of the 1-D model (see equ. (77)).

In the following, we mainly focus on the local magnetization profile in term of $z$ for different values of the reduced external field, $h$.

\section{RESULTS AND DISCUSSION}

We first focus on the magnetization profile obtained at the interface between a cubic grain of magnetically hard material and the soft matrix. We consider a magnetically hard material characterized by $K_{h}=3.0510^{6} \mathrm{Jm}^{-3}, A_{h}=7.710^{-12} \mathrm{~J} / \mathrm{m}^{-1}$ and $J_{h}=1 T$, typical values for the Re-Fe compounds [4], leading to $\delta_{B}=5 \mathrm{~nm}$, the soft matrix being characterized by $K_{s}=0$ and the other parameters defined through the values of $\epsilon_{A}$ and $\epsilon_{J}$. We first compare the demagnetization curves given by the true 3-D model to that one should get from the 1-D profile as calculated from equation (5). The latter is obtained analytically from the magnetization profile and the 
geometrical average weighted by the respective volumes of the hard and soft phases in the 3D model. The result is displayed in figure (1) for 3 characteristic sets of values of $\left(\epsilon_{A}, \epsilon_{J}\right)$. As can be seen, the agreement is qualitatively correct, the main discrepancy being the values of the nucleation and coercive fields. This stems from shape and finite size effect as as been discussed for the nucleation field above. On the other hand, the characteristic plateau in between $h_{n u c l}$ and $h_{c}$ is very well reproduced especially concerning its $h$ - dependence. Then, in figure (2) we compare the magnetization profile $m_{z}(z)=\cos (\varphi(z))$ as calculated in the 1-D description corresponding to $z_{b}^{(s)}=\infty$ to the one obtained from the $3-\mathrm{D}$ simulation. This is done for a set of values of $h$ corresponding to characteristic points on the demagnetization plateau $\left(h_{n u c l}<h<h_{c}\right)$. We see that on the one hand the compression process of the domain wall in the soft side of the interface is clearly evidenced and on the other hand the agreement between the 3-D simulated profile and the 1-D anaytical one is quite satisfactory. The only case where the calculated 1-D magnetization profile deviates from the simulation result at large values of $|z|$ corresponds to a situation $\left(\epsilon_{A}=0.75 ; \epsilon_{J}=0.325\right.$ and $\left.l_{e x}=2.345 \delta_{B}\right)$ where the plateau $m_{x}(z)=-1$ is not reached at the boundary of the system (see figure (2) ) because the value of the field is too small to compress the profile in the limits of the micromagnetic model. We note that the boundary of the system corresponds to $\left|z / \delta_{B}\right|=6.8$. As can be seen in figure (2) the fitting procedure introduced through equation (16) definitely solves the problem. We conclude that the 1-D model leads to a rather good approximation for the magnetisation curve, and a very good approximation for the angular profile at the hard/soft interface. Accordingly the local demagnetization process at the hard grain / soft matrix interface follows the one deduced from the 1-D layered model. In particular, we emphasize that the strong reduction of the coercive field due to the exchange coupling is reproduced although the value of $h_{c}$ differs due to the mentioned shape and size effects resulting from the embedding geometry.

Now we focus on the influence of the edge to edge distance $\Delta$ between two inclusions, still in the parallel orientation, namely $\hat{n}=\hat{x}$ for the two inclusions. The 1-D angular profile is calculated by using the numerical determination of $\varphi_{b}^{(s)}$ as described in section III equation (86). The magnetization profile $m_{x}(z)$ for the value of the field closest to the depinning point of the 3-D simulation is displayed in figure (3). As $\epsilon_{A}$ increases from $\epsilon_{A}=0.162$ to 0.75 , the ratio $(\Delta / 2) / l_{\text {ex }}$ of the distance between the mid-plane and the interface to the exchange length decreases from 2.115 to 0.983 ; hence the flexibility of the profile $m_{x}(z)$ in the soft phase is sufficient for this latter to reach a plateau at $m_{x}(z)=-1$ in between the two hard inclusions only in the first case. Moreover when $(\Delta / 2) / l_{e x} \leq 1.0$ the two inclusions become exchange coupled via the soft phase, and the magnetization reversal occurs as a whole with a one phase like behavior, as can be seen in figure (414) where the corresponding demagnetization curve is displayed. This means that in this case, a strong coupling regime is reached. This is in qualitative agreement with the magnetic phase diagram of Ref. [13] since we clearly evidence the three phases namely the decoupled magnet for $(\Delta / 2) / l_{e x}>2$, the ES coupled magnet for $2>(\Delta / 2) / l_{e x}>1$ and the rigid magnet for $(\Delta / 2) / l_{e x}<1$.

The same conclusions as above hold for the case when one easy axis is not parallel to the interface; Here we have chosen as an exemple $\theta_{h}=\pi / 4$; the 3 -D simulation has been performed only in the two inclusions model with $\theta_{h} \neq 0$ for one of the inclusions. The results for the angular profile $\varphi(z)$ and the magnetization profiles $m_{x}(z)$ and $m_{z}(z)$ are given in figures (5), (6) and (7) respectively. 
One important difference with the case $\theta_{h}=0$ is that the magnetization of the hard phase in the $\theta_{h} \neq 0$ grain presents a reversible variation of $m_{x}$ before switching as is the case in Stoner Wolfarth spherical particles when the easy axis does not coincide with the external field direction. The other difference as already mentioned is the fact that the hard / soft interface for $h<h_{c}$ is a longitudinal (Néel) domain wall instead of transverse (Bloch) one as it is evidenced from the figure (77).

Now as we have shown from the local magnetization profile across the interface that the process for the demagnetization at the layered system holds at the hard / soft interface of 3D hard inclusions in a soft matrix at least for one or two finite sized objects, we relate the demagnetization curve of the two inclusions model to an extended one. We consider a lattice including $N_{p}=256$ cubic inclusions located on the nodes of a simple cubic lattice made of $4(8 \times 8)$ planes and is an extended version of the preceding two grains model. The edge to edge distance between nearest neighbors in each plane is $\Delta=2 \delta_{B}$ as above and the external field is along $\hat{x}$. Two different easy axes distributions have been used; in the first one all the easy axes are close to the $\hat{x}$-axis, with $\Sigma_{i}\left(\left|\left(\hat{n}_{i} \cdot \hat{x}\right)\right|\right) / N_{p}=0.94$ and in the second one the axes are randomly distributed on the unit sphere $\left(\Sigma_{i}\left(\left|\left(\hat{n}_{i} . \hat{x}\right)\right|\right) / N_{p}=0.5\right)$. We have considered the set of parameters as in fig. (4). Since $2 / 3^{-r d}$ of the interfaces are parallel to both the preferential orientation of the $\left\{\hat{n}_{i}\right\}$ and the direction of the applied field, the comparison with the preceding model for the parallel interface is meaningful. As we can see on figure (8]a), at the qualitative level, the demagnetization curve for the easy axes preferentially distributed along $\hat{x}$ is close the that obtained for the 2 - inclusions 'toy' model. The demagnetization curve calculated with the randomly distributed easy axes is displayed on figure ( $8 \mathrm{~b}$ ), and leads to the same type of conclusion but only at a qualitative level. Given the results we have got on the two inclusions 'toy' model, we conclude that the caracteristic plateau in the demagnetization curve is indeed the signature of the local magnetization profile compression / depinning process. However, considering a mesh of the required quality for extracting the magnetization profile in the 256 inclusions model would lead to prohibitively heavy simulations. Indeed, our analysis of the local magnetization profile is based upon a continuous function for the later and its behavior with respect to the external field. Such a requirement from the finite element simulation results, where the profile is obtained from an interpolation between the mesh nodes, can be reached only with a very fine mesh. Nevertheless, on the physical point of view, in spite of its continuous nature the magnetization profile resulting either from an analytical calculation or a finite elements interpolation cannot be interpreted at an infinitely small length scale since the micromagnetic formalism remains a continuous medium type of approach valid beyond some characteristic lenght scale, say $\sim 1 \mathrm{~nm}$.

We conclude that as is the case on the small local model, the rigid magnet, exchange spring and decoupled magnet regimes are reached on the extended model for roughly $\Delta /\left(2 l_{e x}\right)<1,1<$ $\Delta /\left(2 l_{e x}\right)<2$ and $\Delta /\left(2 l_{e x}\right) \geq 2$ respectively. In this case, these boundary values can be related to the volumic fraction $\varphi_{v}$ of the embedded cristallites through $\Delta /\left(2 l_{e x}\right)=\left(\varphi_{v}^{-1 / 3}-1\right)\left(R / l_{e x}\right)$ with the simple cubic geometry. This extended model provides a link with a more realistic modelling of an actual experimental system as was shown in ref. [3]. Thus, the results displayed in figure (8) show that the local demagnetization process explicited at the single inter-grains soft layer can be qualitatively transfered to a realistic situation. 


\section{CONCLUSION}

In this work, focusing on the well established mecanism of exchange coupling between phases in hard / soft composite systems, we made the connection between the demagnetization behavior at the layered system where a 1-D description holds and a fully 3-D composite system. The connection has been done through the local magnetization profile and we have shown that locally at the hard / soft interface this profile can be transfered from the layered to the 3-D systems. While it is well known that the distance between hard objects in a soft matrix as measured w.r.t. the soft phase exchange length is one of the most relevant parameters driving the coupling, this point on the one hand has been made more quantitative and on the hand illustrated by the behavior of the simulated local magnetization profile in a small 3-D model. This latter play the role of an intermediate between the 1-D description of the interface and the true 3-D model.

\section{Acknowledgements}

The numerical micromagnetic simulations were performed using HPC resources from GENSICINES (grant number 2011-096180).

[1] Z. Chen, X. Meng-Burany, and G. C. Hadjipanayis, Applied Physics Letters 75, 3165 (1999).

[2] Z. Chen, X. Meng-Burany, H. Okumura, and G. C. Hadjipanayis, Journal of Applied Physics 87, 3409 (2000).

[3] K. Younsi, V. Russier, and L. Bessais, Journal of Applied Physics 107, 083916 (2010).

[4] G. C. Hadjipanayis, Journal of Magnetism and Magnetic Materials 200, 373 (1999).

[5] E. Kneller and R. Hawig, IEEE Trans. Magn. 27, 3588 (1991).

[6] E. E. Fullerton, J. S. Jiang, M. Grimsditch, C. H. Sowers, and S. D. Bader, Phys. Rev. B 58, 12193 (1998).

[7] R. Victora and X. Shen, IEEE Trans. Magn. 41, 2828 (2005).

[8] R. Skomski, T. A. George, and D. J. Sellmyer, Journal of Applied Physics 103, 07F531 (2008).

[9] D. Suess, Applied Physics Letters 89, 113105 (2006).

[10] D. Suess, Journal of Magnetism and Magnetic Materials 308, 183 (2007).

[11] A. Goncharov, T. Schrefl, G. Hrkac, J. Dean, S. Bance, D. Suess, O. Ertl, F. Dorfbauer, and J. Fidler, Applied Physics Letters 91, 222502 (2007).

[12] A. Aharoni, Phys. Rev. 119, 127 (1960).

[13] G. Asti, M. Solzi, M. Ghidini, and F. M. Neri, Phys. Rev. B 69, 174401 (2004).

[14] G. Asti, M. Ghidini, R. Pellicelli, C. Pernechele, M. Solzi, F. Albertini, F. Casoli, S. Fabbrici, and L. Pareti, Phys. Rev. B 73, 094406 (2006).

[15] M. Ghidini, G. Asti, R. Pellicelli, C. Pernechele, and M. Solzi, Journal of Magnetism and Magnetic Materials 316, 159 (2007).

[16] R. Pellicelli, M. Solzi, V. Neu, K. Häfner, C. Pernechele, and M. Ghidini, Phys. Rev. B 81, 184430 (2010).

[17] A. Y. Dobin and H. J. Richter, Applied Physics Letters 89, 062512 (2006).

[18] R. Skomski and J. M. D. Coey, Phys. Rev. B 48, 15812 (1993).

[19] R. Skomski, Journal of Applied Physics 76, 7059 (1994). 
[20] T. Leineweber and H. Kronmuller, Journal of Magnetism and Magnetic Materials 176, 145 (1997).

[21] H. Kronmüller and D. Goll, Physica B: Condensed Matter 319, 122 (2002).

[22] K. Y. Guslienko, O. Chubykalo-Fesenko, O. Mryasov, R. Chantrell, and D. Weller, Phys. Rev. B 70, 104405 (2004).

[23] F. Casoli, F. Albertini, L. Nasi, S. Fabbrici, R. Cabassi, F. Bolzoni, and C. Bocchi, Applied Physics Letters 92, 142506 (2008).

[24] J.-L. Tsai, H.-T. Tzeng, and B.-F. Liu, Thin Solid Films 518, 7271 (2010).

[25] W. Scholz, J. Fidler, T. Schrefl, D. Suess, R. Dittrich, H. Forster, and V. Tsiantos, Comp. Mat. Sci. 28, 366 (2003).

[26] A. Y. Dobin and H. J. Richter, Journal of Applied Physics 101, 09 K108 (2007). 


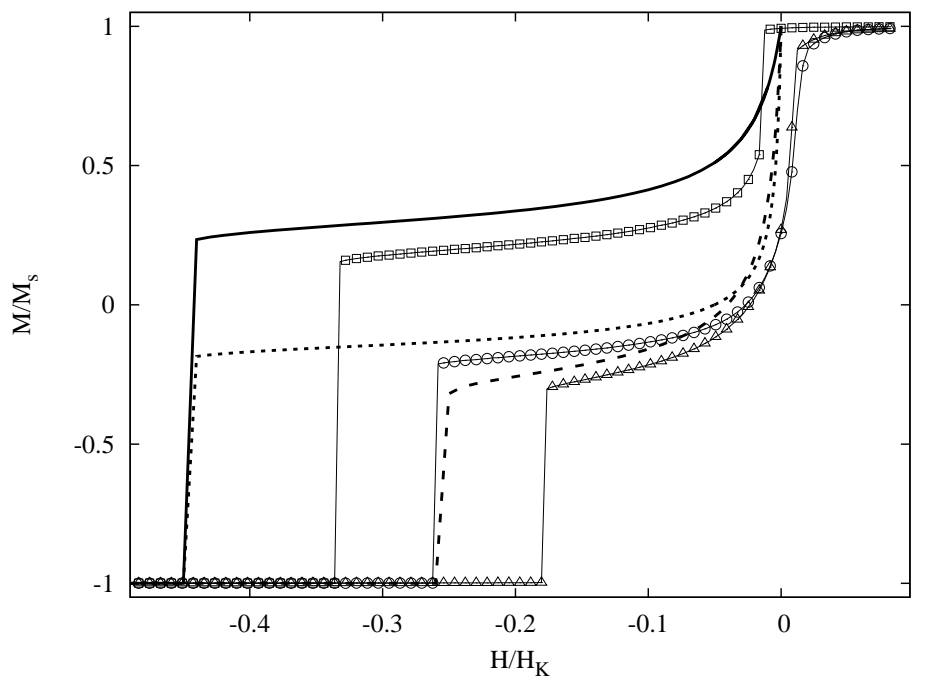

Figure 1: Demagnetization curve as calculated by using the profile calculated on the 1-D model (lines) and compared to the results of the 3 -D micromagnetic simulations (symbols). $\left(\epsilon_{A}, \epsilon_{J}\right)=(0.75,0.325)$ open squares and solid line; $(0.325,0.75)$ open circles and dotted line; $(1.00,1.00)$ triangles and dashed line. 

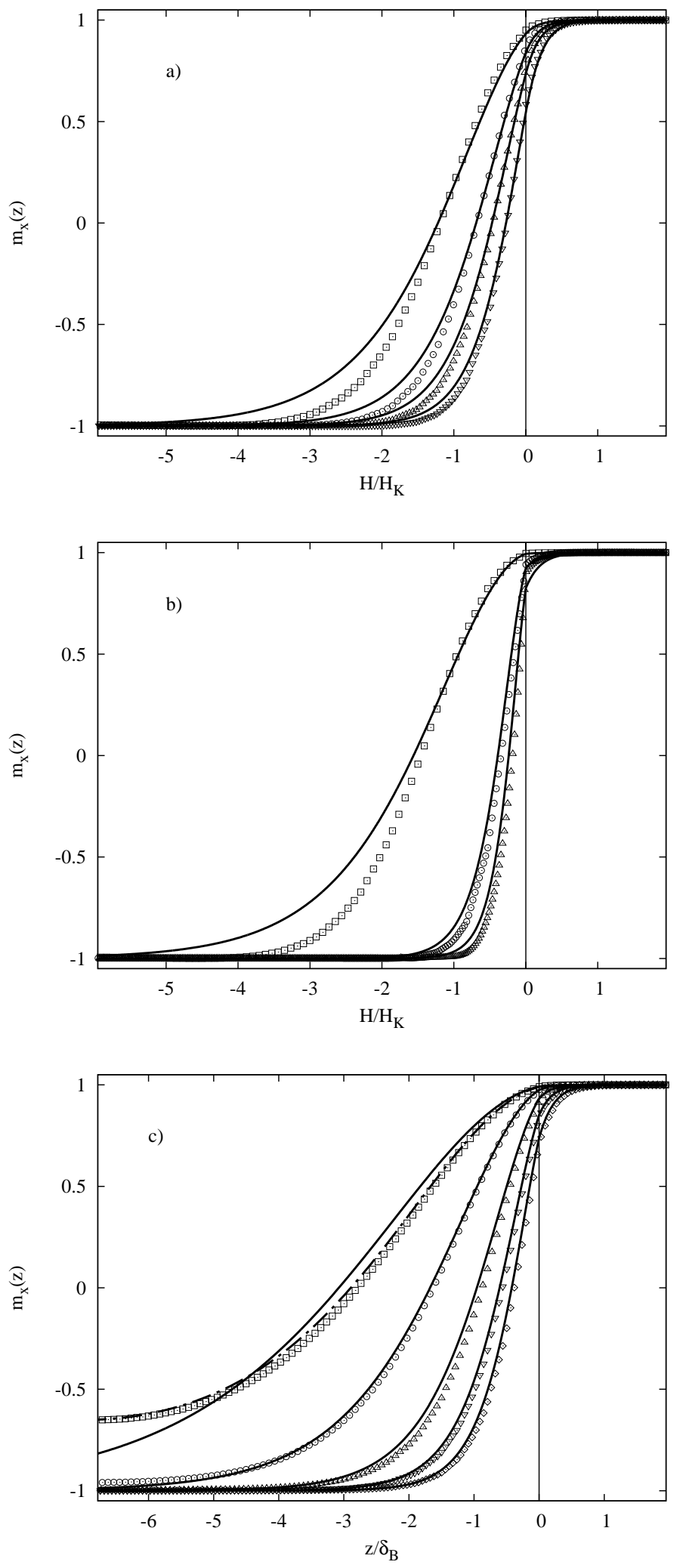

Figure 2: Magnetization profile, $m_{x}(z)$ in terms of $z / \delta_{B}$ across the hard/soft interface along the normal cutting the center of the cubic inclusion. The hard (soft) phase is located at $z>0(z<0)$. Solid line: result of the 1-D model; Symbols: 3-D micromagnetic simulation. $K_{s}=0 ; K_{h}=3.0510^{6} \mathrm{Jm}^{-3}$;

a) : $\epsilon_{A}=1, \epsilon_{J}=1 h=0.0328$, squares; 0.0740, circles; 0.1150, upward triangles; 0.1760, downward triangles. b) : $\epsilon_{A}=0.325, \epsilon_{J}=0.75 h=0.0123$, squares; 0.1352, circles; 0.2580 , triangles. c) $: \epsilon_{A}=$ $0.75, \epsilon_{J}=0.325 h=0.0165$, squares; 0.0492, circles; 0.1230, upward triangles; 0.2295, downward triangles; 0.3320 , diamonds. Dash-dotted line : result of the $1-\mathrm{D}$ model calculated with $z_{b}^{(s)} / \delta_{B}=7.0$ and a fitted value of the field $h^{(f i t)}=0.0240$, according to equation (16). 

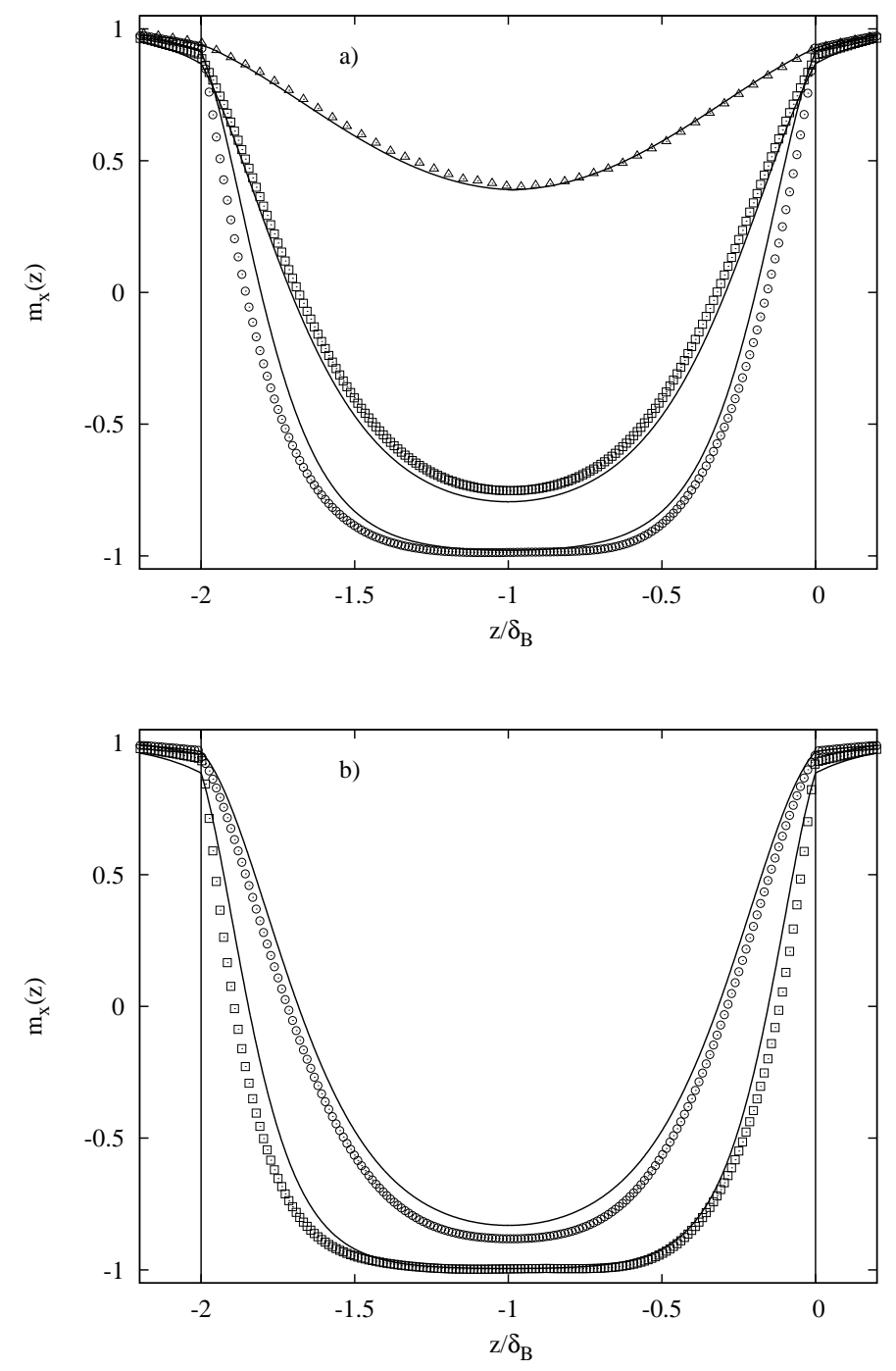

Figure 3: Magnetization profile, $m_{x}(z)$ between the two cubic inclusions in the soft matrix. $\Delta=2 \delta_{B}$. The center of soft layer is located at $z / \delta_{B}=-1.0$. Symbols: 3-D micromagnetic simulation; Solid line: profile as calculated from the 1-D model.

a) $\epsilon_{J}=0.75$ and $: \epsilon_{A}=0.75$ and $h=0.1475$, triangles; $\epsilon_{A}=0.325$ and $h=0.2295$, squares; $\epsilon_{A}=0.162$ and $h=0.2622$, circles. For $\epsilon_{A}=0.75$, the 1-D model profile is calculated with the fitted value of the field, $h^{(f i t)}=0.1874$, according to equation (16). In each case, the value of the field is close to the depinning field of the $3-\mathrm{D}$ micromagnetic simulation.

b) $\epsilon_{J}=1.50 ; \epsilon_{A}=0.1623$ and $h=0.0697$, circes; 0.1885 , squares. The larger value of the field is close to the depinning field of the 3 -D micromagnetic simulation. 


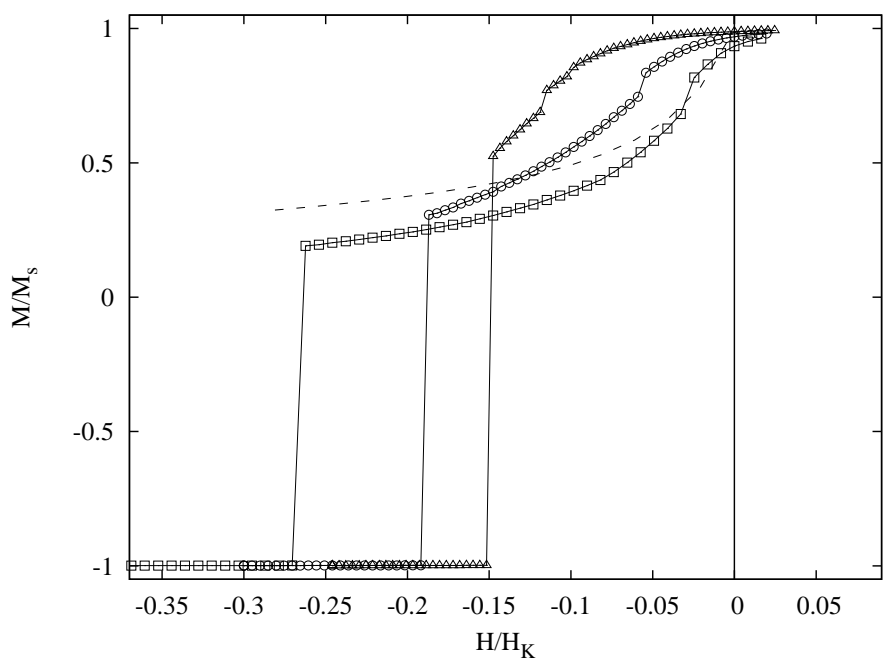

Figure 4: Demagnetization curve of the two cubic inclusions model. $\epsilon_{J}=0.75 ; \epsilon_{A}=0.162$, open squares; 0.325 , open circles; 0.75 open triangles. Dashed line : demagnetization curve of the 1-D model for the hard/soft interface with $\epsilon_{J}=0.75$ and $\epsilon_{A}=0.162$. 


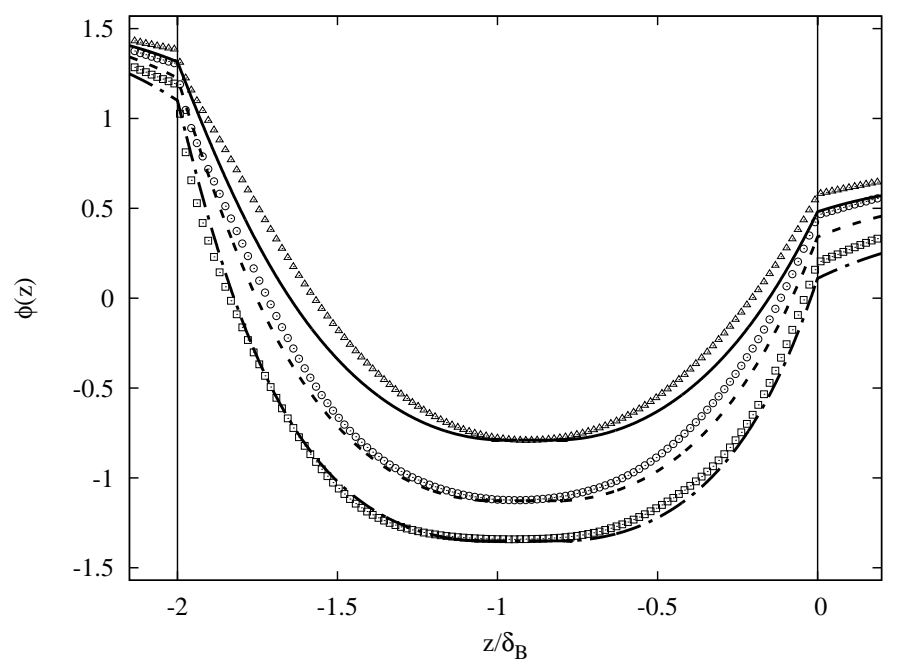

Figure 5: Angular profile accross the soft phase between the two hard cubic inclusions located at $z / \delta_{B}<-2$ and $z / \delta_{B}>0$ respectively with $(\hat{n}, \hat{z})=\pi / 4$ for the latter. Symbols: 3 -D micromagnetic simulations for $h=0.0655$, triangles; 0.1311, circles and 0.3440, squares. 1-D profile for the fitted values of the field $h^{(f i t)}=0.130$, solid lines; 0.2020, dashed lines and 0.3080, dash-dotted lines respectivelly. $h^{(f i t)}$ is determined using equation (16) to fit $\varphi\left(z=z_{b}^{(s)}\right)$ on the simulated result where $z_{b}^{(s)} / \delta_{B}=-1$ is the location of the soft layer mid plane.

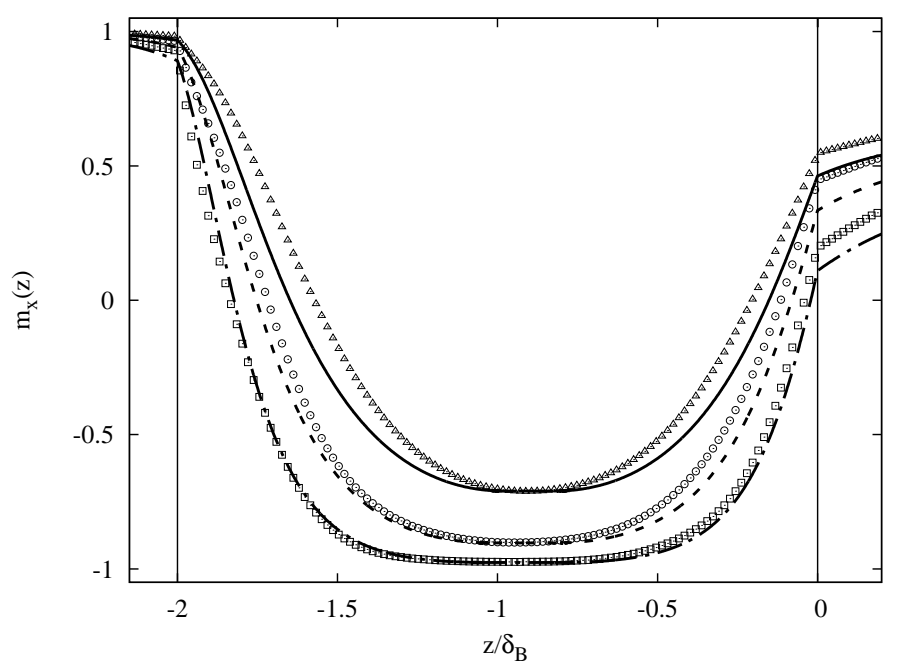

Figure 6: Same as figure (5) for the $x$ - component of the magnetization $\hat{m}(z)$. 


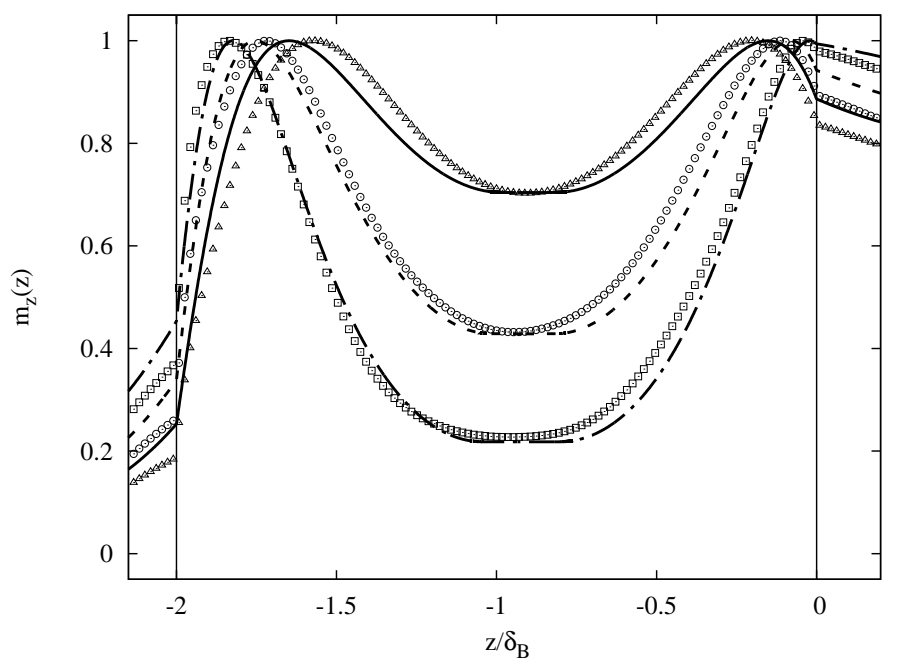

Figure 7: Same as figure (5) for the $z$-component of the magnetization $\hat{m}(z)$. 

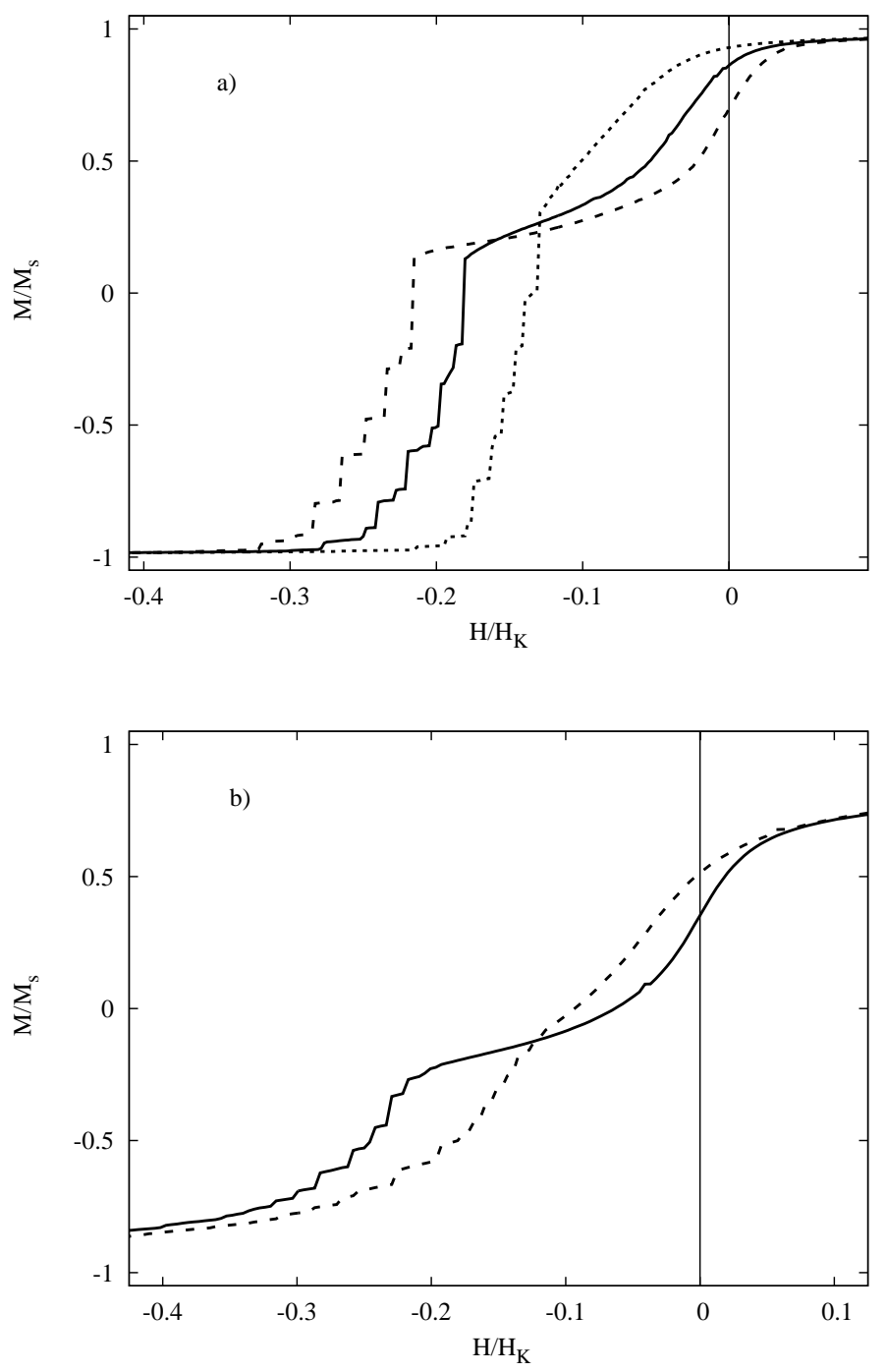

Figure 8: Demagnetization curve obtained from a micromagnetic simulation on the system including 256 cubic particles located on the nodes of a simple cubic lattice.

a) Preferentially oriented easy axes in the direction of the field with $\Sigma_{i}\left(\left|\left(\hat{n}_{i} . \hat{x}\right)\right|\right) / N_{p}=0.94 ; \epsilon_{J}=0.75$ and $\epsilon_{A}=0.162$, dashed line; 0.325 , solid line and 0.75 dotted line.

b) Random distribution of easy axes; $\epsilon_{J}=0.75$ and $\epsilon_{A}=0.162$, solid line; 0.75 , dashed line. 